\title{
Virtual Reality for Learning: Sharing Experiences rather than Resources
}

\author{
Piet Kommers \\ University of Twente \\ Faculty of Behavioral Sciences \\ Division of Educational Instrumentation \\ Kommers@edte.utwente.nl
}

\begin{abstract}
Virtual Reality is becoming a major candidate for embodying immersive learning environments. Whereas in the two preceding decades learning has been conceptualized as situations where students are guided rather than elicited to undertake actions, it is now the right time to explore the other side or the continuum. The tradition of instruction starts from the idea that knowledge and skills need a process of gradual incremental complexity, and thus teaching is the guided tour from initial to finally the execution target performance. At the same time it is a common sense experience that students always build upon earlier experiences and intuition, even if it stems from a slightly different domain.New learning communities rely upon the evidence that learning has both a cognitive, perceptual and social dimension. Virtual Reality allows learning partners to share views, haptics and even allow collaborative experimentation. This workshop will present the more dominant scenarios for Virtual Didactic Laboratories; the needed roles, its mechanisms for self-organisation, diagnostics and final assessment. Demonstrators of VR didactic metaphors will be presented and discussed upon its final implications. The Dime en VRINT projects demonstrate how VR may work out in surgical training. However there are strong indications that the less-advanced 2D shared workspaces have even a more important message here; it is the notion that students need an activity space in order to literally "construct" meanings, concepts and formalities. GRID technologies are not only an answer to the technical needs; they also elicit educational architects to express ideas that went beyond our imagination before. One of them is the challenging idea that learners share spatial models of how they see critical interventions like in medicine, biology and astrophysics; the primitives are objects like human tissues and procedures to be executed. The learning partners will negotiate on best practices seen immediate (visible or tactile) side-effects and thus develop a much more intense apprenticeship than the normal side-looking experience where the expert surgeon just demonstrates his/her best solutions. GRID methodology like proposed collaboratories, and coupled CAVES. The presentation will end up with metaphoric methods to transform educational and training approaches into the arena of GRID-based experiences. Also the projects in the medical domain will be extrapolated into those of engineering, urban planning and art manifestations. Underlying methodology is to map relevant dimensions, scale ontologies for autonomies agents so that learner communities may manifest on self organisation rather than orchestration by the instructor.
\end{abstract}

